\title{
Diseño e Implementación de un Curso para la Formación de Profesores en Matemática: una Propuesta desde la TAD
}

\author{
Design and Implementation of a Course for the Training of Mathematics \\ Teachers: A Proposal since ATD
}

\author{
Ana Rosa Corica ${ }^{*}$ \\ María Rita Otero ${ }^{* *}$
}

\begin{abstract}
Resumen
En este trabajo presentamos y discutimos resultados del diseño e implementación de un curso para la formación de futuros profesores de matemática en didáctica de la matemática. El curso se encuentra fundamentado en la Teoría Antropológica de lo Didáctico, y tiene como propósito que los futuros profesores adopten gestos de un modelo pedagógico no tradicional, basado en la investigación y en la vinculación de la matemática con otras disciplinas. En este trabajo desarrollamos una investigación cualitativa, siendo la metodología propuesta un estudio exploratorio. La investigación se desarrolló en un curso de tercer año de un profesorado en matemática en una universidad nacional Argentina. El curso que diseñamos se compone de dos situaciones. En este trabajo reportamos resultados de la situación dónde se propuso que los futuros profesores estudien una cuestión esencial de la profesión de profesor de matemática, que es: ¿Cómo diseñar e implementar dispositivos didácticos para el estudio de la matemática? Los principales resultados indican que, si bien toda la formación de los futuros profesores estuvo regida por el fenómeno de la monumentalización del saber, logramos incorporar algunos gérmenes de la pedagogía de la investigación y el cuestionamiento del mundo. La comunidad de futuros profesores logró, ante una pregunta sobre la profesión de profesor de matemática, formular y estudiar otras, y que el producto del estudio sea compartido y defendido por todo el grupo.
\end{abstract}

Palabras-clave: Formación de Profesores. Matemática. Teoría Antropológica de lo Didáctico. Recorrido de Estudio e Investigación. Estudio Exploratorio.

\begin{abstract}
In this work, we show and discuss results of the design and implementation of a course about mathematics didactics for mathematics teachers training. The course is based on the Anthropological Theory of the Didactics. It has for purpose that the future teacher adopts a gesture of a non-traditional pedagogy model, based in the research and in the link of mathematics with other disciplines. In this work, we developed a qualitative research, being the methodology proposed an exploratory study. The study was developed at a third year course of a mathematics teacher training at an Argentine national university. The curse has two situations. In one situation, the future teacher had to study an essential question about the teacher profession of mathematics, which is: How do you design and implement didactic devices for the mathematics studies? In this work, we analyzed what gesture of research pedagogy and questioning the world express the future teachers when studied the generating question. The main results indicate that, while all future teacher formation was regulated for the known monumentalism phenome, we incorporated some components of the research pedagogy and questioning the
\end{abstract}

\footnotetext{
"Doctora en Ciencias de la Educación, Universidad Nacional de Córdoba (UNC); Investigador Asistente del CONICET, investigador del NIECyT, Tandil, Buenos Aires, Argentina. Dirección: NIECyT - Facultad de Ciencias Exactas, Pinto 399, 7000, Tandil, Buenos Aires, Argentina. acorica@exa.unicen,edu.ar.

Doctora en Enseñanza de las Ciencias, Universidad de Burgos (UBU); Investigador Independiente del CONICET, investigador del NIECyT, Tandil, Buenos Aires, Argentina. Dirección: NIECyT - Facultad de Ciencias Exactas, Pinto 399, 7000, Tandil, Buenos Aires, Argentina. rotero@exa.unicen,edu.ar.
} 
world. The community of future teachers faced a question about mathematics teacher formation, formulation and other studies. In this manner, the study product was shared and defended by the group.

Keywords: Mathematics Teacher Training. Anthropological Theory of the Didactics. Study and Research Path. Exploratory Study.

\section{Introducción}

Este trabajo se ubica en la problemática de la formación de profesores de matemática. Según CHEVALLARD (2011) la profesión de profesor de matemáticas se encuentra en construcción y debe dotarse de recursos propios de naturaleza matemático-didáctica que constituyan la infraestructura necesaria para afrontar los problemas y desafíos que surgen continuamente en la profesión y que, por su complejidad, el docente no puede abordar de manera individual. La problemática de la formación de profesores no se resuelve mediante propuestas fundamentadas en la descripción del conjunto de competencias necesarias para el ejercicio de la profesión docente. Pues, ello no informa cómo determinar el proceso y las condiciones para adquirir o desarrollar las competencias específicas (BOSCH; GASCÓN, 2009; RUIZ et al., 2014).

En particular, en otra investigación con futuros profesores de matemática, cuya formación se centra en la adquisición de un equipamiento praxeológico didáctico basado en la Teoría Antropológica de lo Didáctico, reportamos que los futuros profesores diseñan y gestionan dispositivos didácticos en los que logran incorporar algunos gestos de la pedagogía de la investigación y el cuestionamiento del mundo que decae en el tiempo, y conduce a que retomen una enseñanza monumentalista. Así, ellos viven el proceso de estudio como el media universal: la introducción de las nociones de estudio es controlada constantemente por el profesor, y los estudiantes son invitados a visitar estos cuerpos de saberes como se visita un monumento que no les es propio. Nuestros resultados evidencian que la formación teórica, no garantiza que los futuros profesores adquieran progresivamente un equipamiento praxeológico didáctico (CORICA; OTERO, 2014).

En este trabajo presentamos resultados parciales del diseño e implementación de un curso para futuros profesores de matemática. En el curso se propone que los futuros profesores adopten un modelo pedagógico basado en la pedagogía de la investigación. En particular, en este trabajo analizamos qué gestos de dicha pedagogía se identifican en un grupo de futuros profesores que estudian una cuestión generatriz esencial de la profesión de 
profesor de matemática, que es: ¿Cómo diseñar e implementar dispositivos didácticos para el estudio de la matemática?

\section{Marco teórico}

Como referencial teórico adoptamos a la Teoría Antropológica de lo Didáctico (CHEVALLARD, 1999, 2007, 2013a, 2013b). La misma plantea la necesidad de introducir en los sistemas de enseñanza procesos de estudio funcionales, donde los saberes no constituyan monumentos que el profesor enseña a los estudiantes, sino herramientas materiales y conceptuales, útiles para estudiar y resolver situaciones problemáticas. Los Recorridos de Estudio e Investigación (REI) son dispositivos que permitirían enfrentar el proceso de monumentalización del saber y hacer vivir lo que Chevallard denomina la pedagogía de la investigación en la clase de matemática.

Para instalar dicha pedagogía, se requiere incorporar un conjunto de gestos didácticos, que implican modificaciones radicales de la enseñanza tradicional: el profesor como el templo del saber, como único garante de la validez de las respuestas, como gestor del tiempo didáctico, y el carácter individual del aprendizaje. Estos elementos quedan sustituidos por el modelo de un proceso de estudio colectivo, dirigido por un profesor que comparte con el grupo de estudiantes la responsabilidad de la gestión de los diferentes momentos didácticos.

El objetivo del estudio viene definido como un conjunto de cuestiones $Q$ vivas a las que la comunidad de estudio se propone aportar una respuesta $R$. Esta respuesta debe constituir en sí misma una aportación significativa, en el sentido de ampliar el universo praxeológico de la comunidad de estudio. En este modelo, durante la actividad de estudio, se movilizarán todos aquellos recursos, medios, saberes y respuestas disponibles que sean necesarios con tal de construir $R$. De esta manera, se acabará generalmente incluyendo praxeologías por lo menos locales, integrando elementos praxeológicos que pueden ir más allá del nivel regional e incluso disciplinario.

En particular, la gestión de una enseñanza por REI requiere ejecutar gestos didácticos, propios del estudio y la investigación, denominados dialécticas (CHEVALLARD, 2007, 2013a, 2013b):

- La dialéctica del estudio y de la investigación. Una investigación supone la combinación del estudio de respuestas preestablecidas, cuestiones y obras, junto a la investigación para pasar de obras precedentes a la respuesta $R$. Consideramos que esta dialéctica constituye el motor de una enseñanza por REI. No es posible 
investigar sin estudiar y a su vez un estudio genuino resulta ser productor de preguntas a ser investigadas.

- La dialéctica del paracaidista y de las trufas. Se refiere a la condición de exploradores que asumen los actores del sistema didáctico. Pues, tienen que tomar una gran distancia del problema y explorar el terreno desde muy arriba. Esta inspección difícilmente encuentra de inmediato lo que se busca, y requiere de gestos de acercamiento, para analizar la utilidad de lo encontrado. Esta dialéctica se opone al hábito escolar de la inmediatez en la búsqueda de soluciones, donde se estudian respuestas inmediatas y triviales.

- La dialéctica de entrar y salir fuera de tema. En un REI se buscan respuestas en sentido fuerte a cuestiones vivas. Es necesario permitir salirse del tema al que inicialmente pertenece la cuestión, incluso hasta de salirse de la disciplina de referencia, para reingresar posteriormente.

- La dialéctica de las cajas negras y cajas claras. Se refiere al proceso de establecer qué conocimiento es pertinente y estudiar mientras se dejan, si es necesario, ciertos saberes en un nivel de gris. Esta dialéctica se opone al hábito escolar que, en general, aspira a una claridad absoluta.

- La dialéctica de la lectura y la escritura. Hace referencia al proceso de evitar la transcripción formal de respuestas existentes. Se trata de tomar de ellas la parte útil y volver a escribirlas en notas de síntesis, glosarios etc. Esta dialéctica se encuentra relacionada con la de las cajas negras y cajas blancas: es preciso establecer con qué nivel de profundidad se transcribirán las respuestas existentes.

- La dialéctica de los media y los medios. La elaboración de las sucesivas respuestas provisionales requiere de respuestas preestablecidas, accesibles a través de los diferentes medios de comunicación y difusión: los media. Los mismos pueden ser libros, artículos de investigación, apuntes de clase etc.

- La dialéctica de la difusión y recepción de respuestas. Se trata del proceso que conduce a difundir y defender la respuesta desarrollada por la comunidad de estudio.

- La dialéctica del individuo y el colectivo. Los estudiantes con su director de estudio deben repartirse el conjunto de tareas y negociar las responsabilidades que debe asumir cada uno. 
- Dialéctica del análisis y la síntesis, praxeológica y didáctica. Para comprender la realidad praxeológica es necesario plantear preguntas didácticas, pero también este análisis tiene un componente epistemológico, que se pregunta por la génesis de las praxeologías en juego.

\section{Metodología}

En este trabajo desarrollamos una investigación cualitativa, siendo la metodología propuesta un estudio exploratorio (HERNÁNDEZ; FERNÁNDEZ; BAPTISTA, 2010). Diseñamos e implementamos un curso fundamentado en la TAD, para el estudio de nociones de Didáctica de la Matemática. El estudio se desarrolló con un grupo de Futuros Profesores en Matemática (FP) que se encontraban realizando el tercer año de una carrera para profesor de matemática.

El curso consta de dos situaciones que son gestionadas de manera simultánea, con el propósito de que los FP adopten un modelo pedagógico no tradicional, basado en la investigación y en la vinculación de la matemática con otras disciplinas. Buscamos integrar la formación matemática de los FP con la formación didáctica, fundamentados en que los FP deben participar de procesos de formación con características similares a las que se pretende que enseñen.

En una de las situaciones, que denominamos enseñanza de la matemática, buscamos que los FP vivan en primera persona el estudio de la TAD involucrados en una enseñanza basada en los principios de la pedagogía de la investigación y el cuestionamiento del mundo. Aquí, se desarrolló una actividad en la que surgieron preguntas no previstas desde un principio, provocando que el estudio se produjera en diferentes direcciones. Abordar con mayor o menor profundidad el estudio de estas cuestiones estuvo relegado al interés de la comunidad. La segunda situación tuvo como objetivo que los FP vivieran un REI codisciplinar desarrollado por Parra, Otero y Fanaro (2013) donde el estudio conduce a establecer vinculaciones entre la matemática y otras disciplinas.

En este trabajo realizamos un análisis pedagógico de la séptima sesión de clase relativa a la situación que identificamos como enseñanza de la matemática. Dicha sesión fue la instancia final del estudio de la cuestión esencial que originó el estudio: $Q_{0}$ : ¿Cómo diseñar e implementar dispositivos didácticos para el estudio de la matemática? En particular, estudiamos las características esenciales del medio de estudio gestado y estudiado FP. 


\subsection{Descripción institucional del curso en el que se desarrolló la investigación}

La investigación se desarrolló en un curso de tercer año de una carrera de profesor en matemática de una Universidad Nacional Argentina. En el programa de estudio del curso se propone formar profesores en la TAD. En cursos anteriores, los FP estudiaron como formación en didáctica de la matemática: la Teoría de las Situaciones Didácticas (BROUSSEAU, 1986), la Dialéctica Instrumento - Objeto y Juego de Marcos (DOUADY, 1988), la Teoría de la Transposición Didáctica (CHEVALLARD, 1985) y los principios fundamentales de la Teoría Antropológica de lo Didáctico (CHEVALLARD, 1999). El curso estaba compuesto por 12 estudiantes, cuyas edades oscilaban entre 20 y 28 años.

El curso duró 4 meses con dos encuentros semanales, uno de 4 horas y otro de 3 horas. Durante los encuentros de 4 horas los FP participaron del REI relativo a enseñanza de la matemática. En estas sesiones el Profesor Investigador (PI) fue el director del estudio. Durante los encuentros de 3 horas, los FP estudiaron un REI codisciplinar. En todas las sesiones los FP formaron los mismos grupos de trabajo. Se conformaron 5 grupos compuestos por 2 o 3 integrantes.

En principio, se firmó un acta de compromiso que se compuso por un conjunto de acuerdos de trabajo para el curso que consensuaron el profesor y los alumnos. Fundamentalmente, esta acta contempla aceptar las respuestas de toda la comunidad de estudio, aceptar el error como parte del aprendizaje, responsabilizarse por su propio aprendizaje, comprometerse en producir y difundir conocimientos y acordar las formas de evaluación del curso.

\subsection{Recolección de datos}

La implementación del REI relativo a la enseñanza de la matemática se realizó en doce sesiones. En todas ellas el PI fue observador participante. Se tomó un audio general de cada sesión y notas de campo antes y durante la implementación de cada una de ellas. También se recogieron todas las producciones de los estudiantes. En la primera sesión, el PI propuso la cuestión generatriz inicial y en las sucesivas clases, cuando las circunstancias lo requirieron proporcionó material para su estudio. Luego de cada sesión los FP completaron un diario de clase on-line que era compartido con la comunidad de estudio. Esto nos permitió recoger las experiencias vividas que manifestaron por escrito los FP. 
En particular, en la primera sesión y como síntesis de las aportaciones de los diferentes grupos de trabajo, la comunidad de estudio elaboró una propuesta global del medio para su estudio durante el curso. Este medio constituyó el punto de partida del REI sobre enseñanza de la matemática, compuesto por los diferentes puntos de vista (CORICA; OTERO, 2015). En las sucesivas sesiones, los grupos aportaron pares de preguntas $\left(Q_{i}\right)$ y respuestas $\left(R_{i}\right)$ según sus intereses y necesidades. El estudio de los pares $\left(Q_{i}, R_{i}\right)$ finalizó en la sesión siete, cuyos resultados se reportan en este trabajo.

\section{Presentación y discusión de resultados}

Para el análisis de datos tomamos como punto de partida la hipótesis de la TAD, según la cual toda actividad humana regularmente realizada puede describirse en términos de praxeologías. Consideramos el conjunto de preguntas propuesto por los FP y las categorizamos según el género de tarea al que remite su estudio. De esta manera, identificamos preguntas que se vinculan con dos géneros: $G^{1}$ : Caracterizar, que se refiere a determinar los atributos de los actores del sistema didáctico (Alumno, Profesor) y las maneras de difundir el saber; y $G^{2}$ : Gestionar, que hace referencia a aspectos relacionados con la dirección o administración del proceso de estudio. Una segunda categorización consistió en identificar los tipos de tareas $\left(T_{j}^{i}\right)$ y tareas $\left(t_{j, h}^{i}\right)$ que requiere el estudio de cada pregunta y que componen los géneros de tareas. Cada pregunta la identificamos como $Q_{l}^{k}$, donde $k$ indica el número de grupo que gestó la pregunta y $l$ identifica a las preguntas gestadas en cada grupo.

En el siguiente esquema (Figura 1) sintetizamos las características del medio de estudio propuesto por los FP.

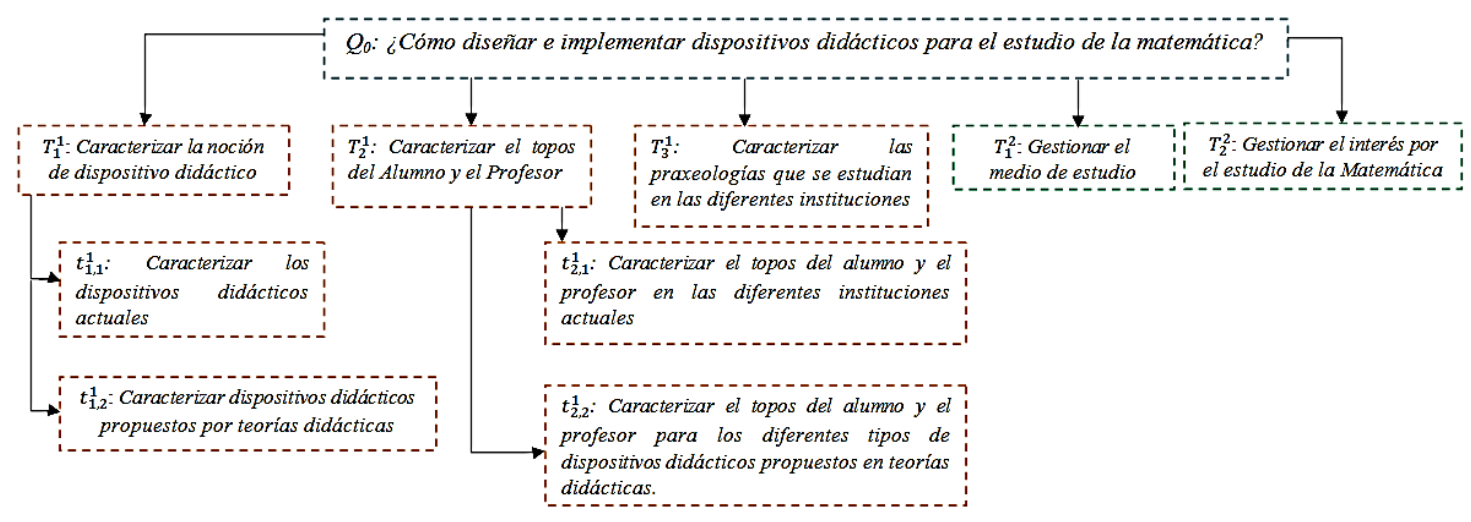

Figura 1- Medio de estudio gestado por los FP

Fuente: Elaboración propia a partir de los protocolos de los FP 
En el esquema se observa que se propone describir cinco tipos de tareas. Tres de ellos se relacionan con $G^{1}$ : Caracterizar, y dos con $G^{2}$ : Gestionar. A continuación profundizamos en las características.

\section{$G^{1}:$ Caracterizar}

$T_{1}^{1}$ : Caracterizar la noción de dispositivo didáctico

Desde el inicio de nuestra propuesta, los FP estuvieron interesados en caracterizar a qué se refiere el término dispositivo didáctico, por lo que se recogieron preguntas representativas de $T_{1}^{1}$. También el interés de los FP estuvo en caracterizar los dispositivos didácticos actuales y los que proponen las distintas teorías didácticas. Estas preguntas se reúnen bajo las tareas $t_{1,1}^{1}$ y $t_{1,2}^{1}$ respectivamente. Las preguntas que reunimos bajo $T_{1}^{1}$ son:

$Q_{1}^{1}$ : ¿Qué es un dispositivo didáctico?

$Q_{1}^{2}, Q_{1}^{3}$ y $Q_{1}^{4}: ¿ Q u e ́$ son los dispositivos didácticos?

$Q_{1,1}^{2}: \vdots$ Es suficiente crear un "buen" dispositivo didáctico para asegurar un aprendizaje exitoso?

$Q_{1,2}^{2}: ¿ Q u e ́$ relaciones y qué diferencias pueden establecerse entre "dispositivo didáctico” y "secuencia didáctica"?

Cuatro grupos acordaron en definir a los dispositivos didácticos como lo sintetizan los estudiantes del Grupo 1: Es la conjunción de procesos y recursos movilizados para producir aprendizajes. El media consultado por los FP para elaborar la definición fue único. Lo constituyó un documento propuesto por el Ministerio de Educación de Paraguay (MEC, 2011). Las raíces etimológicas de la palabra dispositivo marcan sentidos diversos y encontrados (SOUTO et al. 1999). En particular, el filósofo Michel Foucault comprende a los dispositivos como un conjunto heterogéneo de elementos: discursos, instalaciones arquitectónicas, reglamentos e instituciones mediante las que es posible describir las relaciones de poder y de saber (PALACIO, 1998).

Considerando el interés de los FP, hubiese resultado de utilidad establecer discusiones en torno a la noción de dispositivo didáctico y los aportes de diferentes teorías y enfoques en didáctica de la matemática. Esto pone en evidencia que la dialéctica del estudio $e$ investigación es transitada en un solo sentido: la investigación se inicia con la búsqueda de la respuesta. Ante la primera respuesta la búsqueda se detiene, tornándose definitiva e incuestionable.

Por otro lado, los estudiantes del Grupo 2, como respuesta a $Q_{1,1}^{2}$ indicaron que el diseño de dispositivos didácticos es central en la profesión de profesor, pero además destacaron la responsabilidad de los estudiantes en involucrarse en el proceso de estudio: [...] el alumno debe estar predispuesto a involucrarse en la resolución de las tareas. 
Los estudiantes del Grupo 2 diferenciaron entre lo que comprenden por secuencia didáctica y dispositivo didáctico, así aportaron la siguiente respuesta:

Una secuencia didáctica es un [...] plan de clase propuesto por el profesor que pretende alcanzar objetivos concretos y limitados [...] las actividades están vinculadas a un tema [...] y los pasos a seguir están preestablecidos por quien lo diseña [...]. Una situación de aprendizaje debe ser producida por un dispositivo (una organización que disponga de las condiciones necesarias para que ocurra algo) que sitúe al alumno ante una tarea que cumplir, un problema a resolver.

Los estudiantes identificaron diferencias entre dispositivo didáctico y secuencia didáctica, considerando el primero más amplio que el segundo.

$t_{1,1}^{1}$ : Caracterizar los dispositivos didácticos actuales

$Q_{2}^{1}:$ ¿Qué características tienen los dispositivos didácticos actuales en la secundaria?

$Q_{2,1}^{1}$ : En la secundaria, ¿se ofrecen diferentes técnicas para resolver un mismo tipo de tareas?

$Q_{2,2}^{1}:$ ¿Cómo se justifica el uso de técnicas en cierta Institución?

$Q_{3}^{1}:$ ¿Qué características tienen los dispositivos didácticos actuales en la Universidad?

$Q_{1,3}^{4}: ¿ Q u e ́$ características tienen los dispositivos actuales para el estudio de las matemáticas en las diferentes instituciones?

El Grupo 1 caracterizó a la enseñanza actual en la escuela secundaria indicando:

[...] el profesor expone cada saber, sin dar lugar al alumno a ser partícipe activo en el proceso de enseñanza-aprendizaje (...) Los diferentes tipos de tareas estudiadas aparecen poco relacionadas entre sí y se asocia a cada tipo de tareas la técnica que le corresponda, no se le da la responsabilidad al alumno de modificar una técnica conocida para llevar a cabo una tarea algo diferente a la inicial.

Con relación al estudio de la Matemática en el nivel Universitario, en particular destacamos la respuesta aportada por el Grupo 4. Los FP hicieron referencia a los principales resultados reportados por Bosch, Fonseca y Gascón (2004). Este media fue propuesto por el PI. Luego de exponer las dificultades que reportan los investigadores acerca del paso de la Escuela Secundaria a la Universidad, los integrantes del grupo concluyeron en lo siguiente:

Creemos que esto se podría solventar mediante la implementación de los dispositivos didácticos [...] pero existen algunas limitaciones [...] principalmente de los REI, ya que las instituciones no cuentan con la infraestructura necesaria para poder implementarlos [...] Consideramos que [...] la mayoría de los docentes sigue implementando el dispositivo tradicional, donde el profesor presenta los saberes, enseña a resolver ejercicios como una "receta" y luego plantea una serie de estos similares [...] pocas veces se cuestiona la necesidad de justificar la técnica utilizada [...], ni tampoco, cuál es su ámbito de actuación.

La respuesta aportada por ambos grupos no excede los límites del material proporcionado por el PI. No hay búsqueda de otros medias para poder profundizar y caracterizar la enseñanza de la matemática en la Universidad. La respuesta aportada por los FP conduce a preguntarnos: ¿Esta caracterización es propia de todas las instituciones universitarias? ¿Hay propuestas diferentes? Por ejemplo Parra y Otero (2011) desarrollaron 
una investigación sobre la enseñanza de la matemática en el primer año de la Universidad. Las autoras reportan que en esta institución se rompe con el dispositivo tradicional de clases teóricas y prácticas. Aquí los profesores integran lo que ellos comprenden por teoría y práctica. Finalmente, lo que generan es reducir las acciones didácticas al nivel de la praxis. Las clases transcurren sin construir un entorno tecnológico-teórico que dé sentido matemático a las técnicas utilizadas, y el profesor puede ser visto como encerrado en el nivel de las cuestiones que él supone serán evaluadas. Esta investigación, accesible a todo público, pone de manifiesto que hay instituciones que no se rigen por los dispositivos didácticos descriptos por el Grupo 4, más allá de los éxitos y fracasos, provocados por los cambios.

$t_{1,2}^{1}$ : Caracterizar dispositivos didácticos propuestos por teorías didácticas

$Q_{1,1}^{1}: \quad ¿$ Cuáles son los dispositivos didácticos que han surgido para implementarse en la educación matemática?

$Q_{1,1,1}^{1}$ : Cuando se habla de "situaciones del mundo" ¿A qué se hace referencia?

$Q_{1,2}^{1}:$ ¿A qué cuestiones responden los dispositivos didácticos?

$Q_{2,3}^{1}: \vdots$ Cómo modificar (los dispositivos didácticos actuales en la escuela secundaria) en relación a la TAD?

$Q_{3,1}^{1}: ¿$ Cómo modificar (los dispositivos didácticos actuales en la Universidad) en relación a la $T A D ?$

$Q_{6}^{1}:$ Debido a la enseñanza actual los alumnos siempre cuestionan por qué estudiar este tema, para qué sirve, entre otras. Entonces, ¿Cómo permite la implementación de un REI, en la educación matemática, mediante el trabajo autónomo del alumno elucidar estas cuestiones?

$Q_{1,3}^{2}:$ ¿Qué dispositivos didácticos propone la TAD?

$Q_{1,4}^{2}:$ ¿Qué dispositivos didácticos propone la TSD?

$Q_{1,5}^{2}:$ ¿Qué dispositivos didácticos pueden implementarse en la Escuela Secundaria? ¿Y en la Universidad?

$Q_{1,5,1}^{2}$ : ¿Cuáles son las restricciones que operan en cada institución para su plena implementación?

$Q_{1,1}^{3}: \measuredangle C u a ́ l e s$ son los diferentes dispositivos didácticos que se implementan en las distintas instituciones? ¿Qué características tienen?

$Q_{1,2}^{3}: ¿$ Qué limitaciones poseen los dispositivos didácticos antes nombrados?

$Q_{2}^{3}: ¿$ Cómo se debe iniciar el estudio de las matemáticas en la Secundaria?

$Q_{2,1}^{3}: \vdots$ Y en la Universidad?

$Q_{2,2}^{3}: ;$ Siempre se debe comenzar con un problema?

$Q_{7}^{3}: ¿$ Cómo modificar los dispositivos didácticos actuales para mejorar la enseñanza de la secundaria?

$Q_{1,1}^{4}: ¿$ Qué tipos de dispositivos didácticos podemos encontrar?

$Q_{1,2}^{4}: ¿ Q u e ́$ dispositivos nos ofrece la TAD?

$Q_{1}^{5}:$ ¿Qué características tienen los dispositivos didácticos en las diferentes instituciones?

$Q_{1,1}^{5}: ¿$ Cómo gestionar un REI en la escuela secundaria actual? ¿Y en la universidad?

El Grupo 1 para dar respuesta a $Q_{1,1}^{1}$ caracterizó los dispositivos didácticos actuales según una clasificación aportada por GASCÓN (2001). Este media fue propuesto por el grupo de estudiantes, lo que pone de manifiesto los gérmenes iniciales en los FP de la búsqueda propia de medias. Esto resulta ser compatible con vivir la dialéctica del estudio y la 
investigación. Aquí se aportó una respuesta que permitió caracterizar algunos modelos de enseñanza tradicional desde la perspectiva de la TAD. La propuesta de los estudiantes continúo en caracterizar los Talleres de Prácticas Matemáticas (TPM), las Actividades de Estudio e investigación (AEI), los Recorridos de Estudio e Investigación (REI) y las Situaciones Didácticas (SD) a partir del material proporcionado por el PI. En esto último encontramos los mismos rasgos en la respuesta aportada a $Q_{1,3}^{2}$ por el Grupo 2.

Para responder a $Q_{1,4}^{2}$, los FP del Grupo 2 caracterizaron la Teoría de las Situaciones Didácticas (TSD) a partir del material proporcionado por el PI. Por otro lado, el Grupo 3, para dar respuesta a $Q_{1,1}^{3}$, y el Grupo 4, para dar respuesta a $Q_{1,1}^{4}$ y $Q_{1,2}^{4}$, reportaron los dispositivos didácticos propuestos por los Grupos 1 y 2, y caracterizaron la Dialéctica Instrumento Objeto (DI-O). En las respuestas observamos la debilidad del tránsito por la dialéctica de la lectura y la escritura. Pues, los estudiantes realizaron transcripciones formales de respuestas parciales existentes, donde no se cuestiona el texto en el que se las encontró inscriptas, y se toma de ellas solo la parte de su interés. La transcripción de respuesta se encuentra en el nivel que aporta la respuesta inmediata a la pregunta de los FP, sin realizar síntesis y formulación de nuevas peguntas.

Por otro lado, los estudiantes del Grupo 1 buscaron respuesta a la pregunta $Q_{1,1,1}^{1}$. La misma hace referencia a lo que Chevallard se refiere con el estudio de situaciones del mundo. En particular, los integrantes del Grupo 1 fueron los únicos que formularon y respondieron este tipo de pregunta. Los alumnos esbozaron una respuesta, pero no aportaron ejemplos:

Cuando se habla de "situación del mundo" [...] hace referencia a una cuestión inicial que permitirá (...) la incorporación de la razón de ser de las OM curriculares. Las matemáticas son una "situación del mundo" ya que han surgido con la necesidad de dar repuestas a algunas cuestiones planteadas.

En las sesiones posteriores a la que reportamos, se solicitó a la comunidad de estudiantes proponer y estudiar situaciones del mundo. Pues, una de las preguntas que había propuesto el PI en sesiones previas a la que se reporta fue: ¿Qué situaciones del mundo estudiar en las diferentes instituciones? ¿Cómo estudiar estas situaciones del mundo? El propósito fue sumergir a los FP en el estudio de cuestiones vinculadas a la organización de los programas de estudio de matemática. De hecho, la matemática permaneció ausente en todas las preguntas propuestas por la comunidad de estudio. Durante las 7 sesiones iniciales estas preguntas no tuvieron respuesta. Esto hizo que en sesiones posteriores se destinara un período de estudio para la propuesta y análisis de situaciones del mundo. 
En la respuesta a $Q_{1,2}^{1}$ encontramos aportes del Grupo 1 a caracterizar los dispositivos didácticos en función de la TAD y formular preguntas sobre la profesión de profesor en el marco de la TAD:

La creación de dispositivos didácticos es la manera de responder a cuestiones que se plantean al profesor, tales como: ¿Cómo realizar el primer encuentro con una organización matemática? ¿Con qué tipo de tareas?; ¿Cómo conducir el estudio exploratorio en un tipo de tareas dado? ¿Cómo llevar a cabo la institucionalización? ¿Cómo realizar el momento de la evaluación? [...].

La respuesta de los estudiantes continuó indicando las disfunciones de la actividad matemática escolar y las posibles soluciones según los diferentes dispositivos didácticos desarrollados:

[...] la ausencia de un dispositivo didáctico que permita la vida institucionalizada del momento del trabajo de la técnica, esta laguna fue paliada por los TPM. (...) La incompletitud relativa de las organizaciones en el desarrollo (...) es solventada por los AEI. La última de las disfunciones (...) se debe al olvido de la razón de ser de las praxeologías didácticas (...) se intenta solucionar a partir de los REI.

Como respuesta a $Q_{2,3}^{1}$ lo estudiantes del Grupo 1 buscaron dar solución caracterizando los REI como si fuese la posible solución a las problemáticas que hostigan a la educación.

Este tipo de respuesta pone de manifiesto la debilidad de los estudiantes en la vivencia de la dialéctica del estudio y la investigación. Pues la respuesta se aporta a partir del material propuesto por el PI. Los FP no buscaron en otros enfoques en didáctica de la matemática que trasciendan los límites de los media aportados por el PI. Por otro lado, los estudiantes no cuestionaron la factibilidad de la implementación de una enseñanza REI en las condiciones actuales de la escuela secundaria, ni las ventajas y desventajas obtenidas por los investigadores que diseñaron e implementaron REI.

El bajo nivel de investigación de los estudiantes del Grupo 1 se reafirma con el estudio de la respuesta aportada a $Q_{3,1}^{1}$. Los FP caracterizaron a los TPM y REI como dispositivo de posible implementación en la Universidad. Esto condice con las investigaciones desarrolladas en las instituciones. En la literatura científica se puede encontrar reportes de la implementación de estos dos dispositivos en la Universidad, no siendo así para las AEI. Así, el análisis de estos resultados de investigación exceden los límites de la propuesta de los estudiantes.

Por otro lado, como respuesta a $Q_{6}^{1}$, los integrantes del Grupo 1 , nuevamente destacaron la relevancia de una enseñanza por REI. Esta respuesta no fortalece la dialéctica de la lectura y la escritura. La respuesta es una inscripción textual del material aportado por 
el PI. Aquí, no se centra la discusión en la problemática actual de la desfragmentación disciplinar que vive en las instituciones. Una enseñanza por REI requiere de modificaciones profundas de los programas de estudio haciendo vivir el estudio codisciplinar en contraposición del estudio de disciplinas puras que colman los programas de estudios.

Por otro lado, los FP del Grupo 3 como respuesta a $Q_{1,2}^{3}$, indicaron las limitaciones de cada dispositivo:

[...] los TPM no pueden modificar más que localmente la organización didáctica escolar (...) la necesidad de partir de OM puntuales previamente establecidas, que no siempre aparecen como respuestas a cuestiones problemáticas que el grupo de estudiantes se haya podido plantear (....) Dado que las AEI se sitúan en el nivel local no constituye una herramienta eficaz para cuestionar la epistemología escolar monumentalista (...) la actividad del profesor es limitada, sólo tiene la posibilidad de elegir ciertas cuestiones matemáticas que serán estudiadas, no puede ir más allá de los temas curriculares que ya están establecidos (...) Los REI funcionan como un modelo muy general del proceso de estudio, superando las limitaciones de los TPM y de los AEI [...].

Aquí también los FP del Grupo 3 transitaron la dialéctica de la lectura y la escritura de manera deficiente. Los estudiantes aportaron una respuesta donde carece de reflexión de algunos dispositivos didácticos descriptos en $Q_{1,1}^{3}$. En correspondencia con los propuesto por el Grupo 1, el Grupo 3 también sostiene que una enseñanza por REI no tendría limitaciones. Sin embargo, Chevallard (2007) indica que la infraestructura actual de la escuela no se encuentra suficientemente preparada para implementar un REI. Así mismo, el Grupo 3 no menciona limitaciones para una enseñanza por SD y DI-O, pues el material proporcionado por el PI no realiza este análisis reflexivo.

En este mismo sentido, los integrantes del Grupo 2 aportaron respuesta a $Q_{1,5}^{2}$ en donde se evidencia la dialéctica de la lectura y la escritura de manera más eficiente. Aquí, los estudiantes analizan sintéticamente las posibilidades de implementar diferentes dispositivos didácticos que han estudiado durante la formación. En particular, la propuesta del Grupo 2 aporta una respuesta similar a $Q_{1,2}^{3}$ por el Grupo 3, y además hace referencia a la TSD:

Las situaciones didácticas son viables para implementarse en la Escuela porque requieren una responsabilidad por parte del alumno pero sigue siendo el docente quien propone las actividades (...) La evaluación es muy similar a las propuestas en la enseñanza tradicional, sólo que no se considera definitiva en la nota final.

En la respuesta de los estudiantes detectamos interpretaciones propias de la concepción de evaluación en el marco de la TSD. Pues, la teoría no hace referencia a la evaluación en correspondencia con lo propuesto en los sistemas educativos.

Por otro lado, los integrantes del Grupo 2 en referencia a la respuesta de $Q_{1,5,1}^{2}$, para una enseñanza por REI, AEI y TPM indicaron que existirían restricciones temporales que no 
permitirían una plena implementación. Con relación a las SD, nuevamente los estudiantes hicieron referencia al sistema de evaluación de la institución, lo cual consideran incompatible en una enseñanza guiada por la TSD.

Otra de las preocupaciones del Grupo 3 fue preguntar sobre cómo iniciar las clases de matemática $\left(Q_{2}^{3}\right)$. Así, los estudiantes aportaron respuestas que se limitan nuevamente a describir los dispositivos didácticos AEI, TPM, REI y SD.

Como respuesta a $Q_{2,1}^{3}$, el Grupo 3 indicó que se podrían implementar los dispositivos didácticos descriptos en $Q_{2}^{3}$ pero no esbozaron respuestas a la factibilidad de su implementación en diferentes instituciones. En respuesta a $Q_{2,2}^{3}$ los FP retomaron aspectos característicos de los dispositivos detallados y destacaron anomalías de la enseñanza tradicional: “[...] en la actualidad existen clases donde primero se presenta una introducción al tema a enseñar, seguido de una serie de ejercicios o bien problemas generando un saber mecanizado".

Consideramos que los estudiantes no responden a $Q_{2,2}^{3}$.

La respuesta del Grupo 3 a $Q_{7}^{3}$ resulta ser reflexiva de una enseñanza por REI, y que contempla la deficiencia encontrada en el estudio de $Q_{1,1}^{3}$.

Para implementar los dispositivos didácticos en la actualidad (...) se debería tener una consistencia a través de los años, ya que el REI debería continuar aplicándose al siguiente año [...] se necesitaría una mayor participación de la institución y los docentes de los años siguientes.

Los FP aportaron una respuesta que no resulta ser una transcripción textual de medias aportados por el PI. Esto nos hace suponer que los FP transitaron la dialéctica de la escritura y la lectura.

Por otro lado, los integrantes del Grupo 5 para responder a $Q_{1}^{5}$ y $Q_{1,1}^{5}$ caracterizaron la enseñanza en el marco de la TAD en diferentes instituciones:

[...] implementar un REI en la escuela secundaria implica cambios tanto en el sistema como en la formación y el rol del profesor. El alumno [...] debe haber un cambio en su actitud, convirtiéndose en herbartiano, procognitivo, exotérico, enciclopedista ordinario y problematizado. En la universidad, resultaría aún más difícil implementar [...] las clases son multitudinarias, el alumno en muy pocas oportunidades tiene un lugar para construir su propio conocimiento [...]. Aunque las clases tengan un número reducido de alumnos, los profesores no están acostumbrados a darle ese lugar para construir.

A su vez, la respuesta en torno a la enseñanza universitaria se enriqueció señalando las dificultades de una enseñanza por REI en la estructura que separa a las clases en teóricas y prácticas.

$T_{2}^{1}$ : Caracterizar el topos del alumno y el profesor 
Los 5 grupos formularon pares de $Q$ y $R$ para caracterizar el topos del alumno y el profesor en el proceso de estudio de la Matemática. Clasificamos las preguntas según buscan caracterizar el topos del alumno y el profesor en las instituciones actuales $\left(t_{2,1}^{1}\right)$ y lo propuesto en teorías didácticas $\left(t_{2,2}^{1}\right)$.

$t_{2,1}^{1}$ : Caracterizar el topos del alumno y el profesor en las diferentes instituciones actuales

$Q_{6}^{2}: ¿ Q u e ́$ diferencia hay entre un profesor de nivel Secundario y Universitario?

$Q_{6,1}^{2}: ; Y$ en los alumnos?

$Q_{4}^{3}: ¿$ Cuál es la relación entre el docente y el alumno en la Secundaria? ¿Y en la universidad?

Los estudiantes del Grupo 2 aportaron respuesta a $Q_{6}^{2}$ unificando las características de los profesores de la escuela secundaria y la universidad: "Los docentes de ambas instituciones en la enseñanza tradicional, su trabajo se enfoca en presentar contenidos a los estudiantes, a los cuales se espera que "respondan" adecuadamente al momento de ser evaluados".

Con relación al topos del alumno, el Grupo 2 indicó: "En la Escuela se considera al alumno pasivo (...) Su protagonismo aumenta [...] en la teoría de las Situaciones Didácticas o de la teoría Antropológica de lo Didáctico [...]”.

Los estudiantes caracterizaron a estudiantes universitarios y de secundario, así como su topos para las teorías didácticas estudiadas. Esto fue retomado en las respuestas a las preguntas que componen a $t_{2,2}^{1}$. Los integrantes del Grupo 3 aportaron respuesta a $Q_{4}^{3}$ de manera similar a la propuesta del Grupo 2.

$t_{2,2}^{1}$ : Caracterizar el topos del alumno y el profesor para los diferentes tipos de dispositivos didácticos propuestos en teorías didácticas

$Q_{1,3,4}^{1}:$ ¿Cuál debería ser el topos del alumno y cuál el del profesor?

$Q_{3,2}^{1}:$ ¿Cuál debería ser el topos del alumno y cuál el del profesor [en relación a la TAD]?

$Q_{4}^{1}: ¿$ Cómo influye el trabajo autónomo de los alumnos en una Organización Didáctica?

$Q_{4,1}^{1}: ¿$ Qué ocurre si el trabajo del alumno no es totalmente autónomo?

$Q_{5}^{1}: ¿ Q u e ́$ hacer cuando el alumno no dispone de los conocimientos previos necesarios para aprender el conocimiento matemático en cuestión?

$Q_{3}^{2}$ : ¿Cómo participan los actores del sistema didáctico en los diferentes dispositivos didácticos?

$Q_{1,3}^{3}: ¿$ Cuál debería ser el topos del alumno y del profesor en cada uno de los dispositivos didácticos?

$Q_{4,1}^{3}: ¿$ Cómo debería ser según la TAD [la relación entre alumnos y profesores]?

$Q_{4,2}^{3}: \measuredangle Q u e ́$ lugar ocupa la Institución en esto [la relación entre alumnos y profesores]?

$Q_{4,3}^{3}: ¿$ ¿Cuál es la dificultad didáctica más común para el profesor?

$Q_{5}^{3}:$ :De qué forma se puede desarrollar una clase cuando los alumnos van a diferente ritmo de aprendizaje?

$Q_{2}^{4}: \vdots$ Qué características deben tener los problemas y las actividades que se estudian en las diferentes instituciones?

$Q_{2,1}^{4}: ¿$ Quién las propone? ¿Cómo elegirlas? 
$Q_{3}^{4}:$ ¿Cuál es el rol del profesor y el rol del alumno en la clase de matemáticas según la TAD y su interacción con el saber?

$Q_{3,1}^{4}: \measuredangle Q u e ́$ ocurre en las diferentes instituciones [en relación al rol del profesor y el alumno]?

$Q_{4}^{4}:$ ¿Cómo debe hacer el profesor para despertar la razón de ser o el sentido de las OM estudiadas en los alumnos?

$Q_{4,1}^{4}:$ ¿Cómo es en la escuela y cómo es en la universidad?

$Q_{5}^{4}: ¿$ Cómo lograr el equilibrio entre la diversidad de alumnos?

$Q_{6}^{4}: ¿$ De qué manera el alumno va a construir un nuevo conocimiento según la TAD?

$Q_{2}^{5}: ¿$ Cómo lograr el equilibrio en una clase en la que los alumnos no disponen de los mismos conocimientos previos?

$Q_{2,1}^{5}: ¿$ Qué hacer cuando el alumno no dispone de los conocimientos previos que necesita?

$Q_{4}^{5}$ : ¿Cómo es la interacción entre alumno y profesor en las diferentes instituciones?

$Q_{4,1}^{5}: ¿$ Cómo debería ser la interacción entre alumno y profesor en las diferentes instituciones para la TAD?

$Q_{5}^{5}: ¿ C u a ́ l$ es la razón de ser de las praxeologías que se estudian en las diferentes instituciones?

$Q_{5,1}^{5}: ¿$ Cómo hacer para que los estudiantes vivan la razón de ser de la matemática que se estudia en las diferentes instituciones?

Como respuesta a $Q_{1,3,4}^{1}$ los integrantes del Grupo 1 esbozaron una respuesta general sobre el lugar del alumno y el profesor en el proceso de estudio:

El profesor debe crear un topos apropiado que dé al alumno el sentimiento de tener un "verdadero papel que desempeñar" [...]. El alumno debe aceptar al profesor como un director de estudio, y al mismo tiempo, renunciar a las engañosas facilidades que le ofrece como profesor.

Por otro lado, los integrantes del Grupo 1, para dar respuesta a $Q_{3,2}^{1}$ aportaron una respuesta focalizada en una enseñanza por TPM. Esta respuesta ignora los AEI, REI y las SD, que son los dispositivos didácticos discutidos por la comunidad de FP. En la respuesta, los estudiantes caracterizaron al profesor de un TPM como el responsable del diseño de las tareas a estudiar, las técnicas y el entorno tecnológico teórico. A los estudiantes se les asigna la tarea de responsabilizarse en los momentos del trabajo de la técnica y la evaluación. En particular, al responder $Q_{4}^{1}$, enfatizaron el trabajo autónomo de los alumnos: “[...] cuanto mayor sea el trabajo por sí solo del alumno mayor será el aprendizaje que este adquiera, dado que si el alumno trabaja por su cuenta puede construir su conocimiento".

Aquí, los estudiantes propusieron una respuesta única y directamente proporcional entre la cantidad de trabajo autónomo y los aprendizajes realizados. Pues, como respuesta a $Q_{4,1}^{1}$, los estudiantes problematizaron qué ocurre si los alumnos no son autónomos para el estudio:

Cuando el trabajo del alumno no es totalmente autónomo se pierde o se limita la posibilidad de que esté construya conocimiento por sí sólo. Formando un conocimiento mecánico o memorístico y cuando se pasa la etapa de la evaluación todo el conocimiento se habrá olvidado. 
Los estudiantes del Grupo 3 (como respuesta a $Q_{4,1}^{3}, Q_{4,2}^{3} Q_{4,3,1}^{3}$ ), el Grupo 4 (como respuesta a $Q_{3,1}^{4}$ ) y el Grupo 5 (como respuesta a $Q_{4}^{5}$ ) ofrecieron respuestas similares a la aportada por el Grupo 1.

Por otro lado, en la misma temática, los estudiantes del Grupo 2 respondieron $Q_{3}^{2}$ considerando que: "Tanto alumnos, profesores y objetos de estudio tienen una participación activa en los dispositivos propuestos por la TSD y la TAD”.

En general, los FP consideran que el alumno debe responsabilizarse del estudio de los problemas y el profesor es considerado como un ingeniero cuya actividad se centra en diseñar dispositivos didácticos, que se encuentran en estrecha relación con el diseño curricular. Estas mismas ideas se recogen del Grupo 4 en respuesta a $Q_{2}^{4}$ y $Q_{1,1}^{4}$ y $Q_{4}^{4}$. En particular, los Grupos 4 y 5 ofrecieron respuesta señalando la problemática del estudio de la OM en las distintas instituciones, e indicando la problemática de la pérdida de razón de ser del estudio de las $\mathrm{OM}$, proponiendo el estudio de problemas con las siguientes características, según Barquero (2009):

- Los problemas deben provenir de cuestiones que la Sociedad propone que se estudien en la institución.

- Los problemas deben aparecer en ciertas cuestiones umbilicales, es decir cuestiones que vinculan con aspectos del mundo y generan nuevas cuestiones. Deben estar situadas en la raíz central de la matemática.

- Los problemas deben estar relacionadas con otras cuestiones que se estudian en la escuela, ya sea de matemática o de otra disciplina.

Aquí se aportó una respuesta desde un media propuesto por el Grupo 4.

Como respuesta a $Q_{1,3}^{3}$ el Grupo 3 destacó el topos del alumno y el profesor para dispositivos desarrollados en el marco de la TAD. Así, para estos estudiantes también el topos del profesor es vivido como el de un ingeniero. En particular, para una enseñanza por REI indican que:: el trabajo es conjunto, desplazando así, el actor individual. Para estos FP [...] los alumnos deberían tener un papel destacado en la propuesta de las cuestiones estudiadas. En particular, el Grupo 4 sostiene que en una enseñanza por REI el profesor como el alumno comparten la responsabilidad de la topogénesis, cronogénesis y mesogénesis.

El Grupo 5, como respuesta a $Q_{4,1}^{5}$, indicó que:

Según la TAD, en la escuela, las tareas deben ser cooperativas [...]. Una de las dificultades [...] para el profesor es "dar un lugar a los alumnos" [...] Según la Pedagogía de la Investigación y el Cuestionamiento del Mundo (PICM) no debe ser una dificultad para el proceso de enseñanza que el alumno no disponga de los conocimientos previos, ya que debe adoptar una actitud procognitiva [...] Esta postura es adoptada por los REI ya que son un dispositivo preparado para que los estudiantes tengan una participación activa, tanto con el profesor como con el saber. 
Por otro lado, los integrantes del Grupo 3 al responder $Q_{5}^{3}$, nos conducen a inferir que tuvo lugar la dialéctica de la lectura y la escritura. Pues aportaron una respuesta que no se reduce a una transcripción textual de algún media:

A la hora de desarrollar una clase según los REI, el docente permite que los alumnos vayan a su propio ritmo, ya que a cada uno a partir de una cuestión generatriz le surgen diferentes cuestiones y respuestas parciales, que ellos mismo van a decidir cuando responder.

$T_{3}^{1}$ : Caracterizar las praxeologías que se estudian en las diferentes instituciones

Aquí, reunimos las preguntas que dan respuesta a cómo describir las praxeologías que se estudian en las instituciones. Se tratan de preguntas que nacen del estudio del media propuesto por el PI. No surgieron de manera natural de la comunidad de FP a partir de $Q_{0}$.

$Q_{2}^{1}:$ ¿Cómo estudiar una praxeología matemática?

$Q_{1,2}^{5}: ¿$ ¿Cómo describir una organización matemática? ¿Por qué existe?

$Q_{1,3}^{5}:$ ¿Cómo describir una organización didáctica?

$Q_{1,4}^{5}:$ ¿Qué relación existe entre estas (Organizaciones Matemáticas y Didácticas)?

Como respuesta a $Q_{2}^{1}$ el Grupo 1 esbozó una respuesta que reflexiona sobre las OM que se estudia:

Estudiar una cuestión conduce a crear, es decir, elaborar una praxeología. Pero, en la escuela, estudiar una cuestión es casi siempre recrear para sí mismo y para sus compañeros de estudio una respuesta ya producida en cualquier otra institución.

Así mismo, la respuesta de los estudiantes se extendió a indicar que el estudio de una praxeología matemática requiere del tránsito de los 6 momentos de estudio señalados por CHEVALLARD (1999). El Grupo 5 focalizó la atención en cómo describir una OM y OD. Así, la propuesta se centró en definir una OM, una OD y caracterizar los momentos didácticos. Como respuesta a $Q_{1,4}^{5}$ los FP destacaron: "Se trata de dos aspectos inseparables ya que no hay organizaciones matemáticas sin un proceso de estudio que las genere, pero tampoco hay un proceso de estudio sin organizaciones matemáticas en construcción".

$G^{2}:$ Gestionar

$T_{1}^{2}$ : Gestionar el medio de estudio

Aquí se reúne preguntas que procuran establecer cómo gestionar el estudio de problemas propuestos por los alumnos.

$Q_{3}^{1}: ¿ Q u e ́$ hacer cuando los alumnos traen problemas a la clase que se alejan del dispositivo didáctico planteado?

$Q_{2}^{2}: ¿$ Quién o quiénes son los responsables de elegir las actividades para los alumnos y con qué criterios?

$Q_{2,1}^{2}: \measuredangle Q u e ́$ objetivos se persigue con esas actividades?

$Q_{5}^{2}: ¿ Q u e ́$ hacer cuando los alumnos llevan problemas a la clase?

$Q_{6,1}^{4}: ¿$ Cómo "testear" los conocimientos previos de los alumnos para abordar los nuevos conocimientos? 
$Q_{6,2}^{4}: \vdots$ Cómo hacer cuando los alumnos no disponen de los conocimientos previos para abordar un nuevo conocimiento matemático?

$Q_{3}^{5}: ¿ Q u e ́$ hacer cuando los alumnos traen problemas a la clase y son ajenos al contenido matemático que se está estudiando?

Una de las problemáticas centrales que señalaron 3 grupos sobre la profesión de profesor es qué hacer cuando los alumnos traen problemas a la clase $\left(Q_{3}^{1}, Q_{5}^{2}, Q_{3}^{5}\right)$. Los FP destacaron la importancia de que los estudiantes realicen preguntas en el marco de una enseñanza por SD, AEI, TPM y REI.

Por otro lado, los integrantes del Grupo 1 aportaron una respuesta resaltando la importancia de que los estudiantes realicen preguntas. Elaboraron una respuesta que excede el material propuesto por el PI:

Es muy común que cuando los alumnos realizan alguna pregunta o formulan algún problema, que puede estar relacionado o no con el tema que se están estudiando [...] que el profesor conteste ahora no es el momento, cuando veamos eso te lo explico. Esto no le da la posibilidad al alumno que construya conocimiento, porque solo lo restringe al conocimiento que se está tratando pero no se puede ir más allá. También fomenta en los alumnos la idea errónea que no deben hacer preguntas; lo cual permite formar personas muy poco cuestionadoras. El profesor en este caso se asegura de proponer un recorrido perfectamente preestablecido, es decir presenta la obra a la cual nadie puede cuestionar y solo deben observar $[\ldots]$

Los estudiantes del Grupo 2 ofrecieron respuestas a $Q_{2}^{2}$ y $Q_{2,1}^{2}$ que caracterizan quién propone el medio de estudio en las clases: "Normalmente, es el profesor el único responsable de diseñar las actividades que presentará en el aula o de evaluar si son adecuadas o no las que ya se hayan formulado".

Como respuesta a $Q_{6,1}^{4}$ los estudiantes del Grupo 4 indicaron: "No conocer los conocimientos previos que poseen los alumnos, no representa un obstáculo dentro de la TAD, ya que propone adoptar una postura procognitiva $[\ldots]$ ".

Por otro lado, como respuesta a $Q_{6,2}^{4}$ los estudiantes indicaron:

Dentro de la concepción constructivista [...] "El factor más importante que influye en el aprendizaje es lo que el alumno ya sabe. Averígüese esto y enséñele en consecuencia." (AUSUBEL; NOVAK; HANESIAN, 1983). [...] Al iniciar un proceso de enseñanza aprendizaje, es necesario analizar, explorar cual es el contenido básico sobre el cual se centrará el proceso. Se debe considerar los objetivos concretos en relación al contenido que se quiere abordar [...]. En el caso de que los alumnos no posean los conocimientos previos necesarios [...] van a tender al aprendizaje memorístico y poco significativo [...] Al detectar conocimientos previos inexistentes, erróneos [...] una medida válida sería reconsiderar los objetivos que se persiguen [...] Los dispositivos propuestos por la TAD (los REI específicamente) movilizan el sistema cognitivo de los alumnos y evocan los conocimiento preexistentes.

Aquí, encontramos una respuesta en la que los estudiantes salen del tema para aportar una respuesta desde una teoría cognitiva. Consideramos que esta teoría no es compatible con 
una enseñanza por REI. En la Teoría de Aprendizaje Significativo resulta vital saber sobre los conocimientos de los estudiantes: "El factor más importante que influye en el aprendizaje es lo que el alumno ya sabe. Averígüese esto y enséñese consecuentemente" (AUSUBEL, 1976, p. 6). En una enseñanza por REI no interesa con tanto énfasis lo que los estudiantes saben, pues se procura formar ciudadanos procognitivos y exotéricos.

$T_{2}^{2}$ : Gestionar el interés por el estudio de la Matemática

Este tipo de tarea se compone por una pregunta: $\mathrm{Q}_{4}^{2}: \measuredangle$ Cómo despertar interés de estudiar Matemática en los alumnos?

Para dar respuesta a $Q_{4}^{2}$ los integrantes del Grupo 2, en primer lugar listaron las posibles causas de desinterés de los estudiantes en el estudio de la matemática, tal como distracción en el aula, la matemática es difícil, no poder abordar problemas matemáticos, no les gusta estudiar, las clases expositivas. Por otro lado, los estudiantes responsabilizaron al profesor de generar interés indicando:

Para generar interés y motivación en los alumnos, es necesario que el profesor utilice todos los recursos de los que dispone [...] resolución de problemas, enseñarles estrategias para la comprensión de ideas [...]. Implementar herramientas tecnológicas [...]. El trabajo en el aula debe ser interactivo [...]. Hacer énfasis en la importancia de la actividad matemática en la sociedad y concientizarlos de que es una herramienta para la vida diaria, que tengan la posibilidad de preguntar sobre cuestiones abiertas que puedan dar lugar a otras nuevas.

Si el aprendizaje matemático se desarrolla a partir de situaciones del mundo [...] tales situaciones pueden interpretarse a través de un modelo matemático con el cual pueden sacar conclusiones.

La respuesta aportada resulta del estudio del media aportado por el PI. En particular, a pesar de todas las discusiones detectamos la fuerte designación de responsabilidad al profesor y de su figura monumental, en particular cuando los estudiantes indican: enseñarles estrategias para la comprensión de ideas.

\section{Comentarios finales}

Presentamos resultados del medio de estudio gestado por los FP para su formación en didáctica de la matemática. Durante las primeras 7 sesiones, los FP fortalecieron la necesidad de formular preguntas, que van más allá de la simple demanda de información. El medio gestado y estudiado por los FP contiene una gran proporción de preguntas centradas en caracterizar los atributos de los actores del sistema didáctico (Alumno, Profesor) y las maneras de difundir el saber. 
En particular, consideramos que la dialéctica del estudio y la investigación fue transitada de manera débil, pues los FP formularon preguntas, pero ante la primera respuesta finalizaron su búsqueda. Una genuina búsqueda de respuestas genera nuevas preguntas, que la comunidad de estudio debe decidir cuándo y de qué manera responder. Esta debilidad generó la deficiencia en la gestión de las restantes dialécticas. Así, por ejemplo, la dialéctica de los medias y los medios fue vivida prácticamente por el PI.

Con relación a la dialéctica del paracaidista y de las trufas fue transitada a lo largo de las siete sesiones con cierta tensión por los FP. Pues resultó difícil controlar la ansiedad de la búsqueda de respuestas inmediatas y acabadas, siendo que el producto relativamente final del estudio se obtuvo luego de dos meses.

Por otro lado, con relación a la dialéctica de entrar y salir de tema no fue gestionada. Los FP se centraron en dar respuesta a partir del media proporcionados por el PI y los aportados por los FP que no se alejan de la propuesta del PI. La comunidad no formuló cuestiones que requieren salirse del tema.

La dialéctica de las cajas negras y claras no fue vivida con total plenitud. Como ocurre en la enseñanza tradicional, los FP buscaron dar una claridad total a las preguntas que estudiaron. Se propusieron respuestas pormenorizadas y especializadas de los dispositivos didácticos que se proponen en el marco de la TAD y TSD. Esto hizo que se formularan pares de preguntas y respuestas, sin formular nuevas preguntas que no encuentran respuestas en la TSD y la TAD.

Con relación a la dialéctica de la lectura y la escritura detectamos algunos gérmenes que hacen suponer su vivencia. En general el foco de atención de los FP estuvo en la transcripción textual de respuestas. Esto requirió de constantes intervenciones del PI para que los FP, a lo largo de las siete sesiones, formularan preguntas y respuestas que condujeran a la elaboración de síntesis.

La dialéctica de la difusión y recepción fue transitada de manera fuerte porque las respuestas aportadas fueron producto de la actividad gestada en toda la comunidad de estudio. Así también, en el programa de estudio tuvo lugar la dialéctica del individuo y el colectivo. Pues aquí se demarcaron los lugares de los integrantes de la comunidad. La responsabilidad del estudio no recayó en el individuo, sino en la comunidad productora, que sostuvo y validó las respuestas que generó colectivamente.

Concluimos en que lo que gestamos en un principio como REI no fue vivido como tal. Pues el estudio de $Q_{0}$ hizo que solo se reconstruyeran respuestas en función del media proporcionado por el PI. A lo largo del estudio logramos incorporar algunos gérmenes de la 
pedagogía de la investigación y el cuestionamiento del mundo. La comunidad de FP, que se caracterizan por haber vivido una formación regida por el fenómeno de la monumentalización del saber, logró ante una pregunta formular otras en vez de respuestas, y que el producto del estudio sea compartido y defendido por todos los FP.

Nuestras investigaciones se orientan a modificar el programa de estudio estableciendo innovaciones en la cuestión generatriz inicial y su gestión. Proponemos involucrar a los FP de matemática, en el estudio de la cuestión central de su profesión que es: $Q:$ ¿Cómo enseñar Matemática? Esta cuestión permitirá evitar a la problemática que nos enfrentamos al proponer $Q_{0}$. Pues los FP, en primer lugar, orientaron sus pares de preguntas y respuestas a definir qué es un dispositivo didáctico. Consideramos que el estudio de $Q$ generaría un conjunto de pares de preguntas y respuestas que trasciende la didáctica y que su estudio permitiría relacionar las disciplinas que, de manera desfragmentada, estudian los FP en su carrera, tales como psicología, epistemología y matemática.

\section{Referencias}

AUSUBEL, D. Psicología Educativa. Un punto de vista cognoscitivo. México: Trillas, 1976.

AUSUBEL, D.; NOVAK, J.; Y HANESIAN, H. Psicología Educativa. México: Trillas, 1983.

BARQUERO, B. Ecología de la modelización matemática en la enseñanza universitaria de las matemáticas. 2009. 564f. Tesis (Doctorado en Matemáticas) - Departament de Matemàtiques, Universitat Autònoma de Barcelona, Bellaterra, 2009.

BOSCH, M.; FONSECA, C.; GASCÓN, J. Incompletitud de las organizaciones matemáticas locales en las instituciones escolares. Recherches en Didactique des Mathématiques, Grenoble, v. 24, n. 23, p. 205-250, 2004.

BOSCH, M.; GASCÓN, J. Aportaciones de la Teoría Antropológica de lo Didáctico a la formación del profesorado de matemáticas de secundaria. In: SIMPOSIO DE LA SEIEM, $13^{\text {th }}$, 2009, Santander. Actas... Santander: SEIEM, 2009, v. 1, p. 89-113.

BROUSSEAU, G. Fondements et méthodes de la didactiques des mathématiques. Recherches en Didactique des mathématiques, Grenoble, v. 7, n. 2, p. 33-115, 1986.

CHEVALLARD, Y. La transposition didactique. Grenoble: La Pensée Sauvage, 1985.

CHEVALLARD, Y. L'analyse des pratiques enseignantes en théorie anthropologique du didactique. Recherches en Didactique des Mathématiques, Grenoble, v. 19, n. 2, p. 221-266, 1999.

CHEVALLARD, Y. Passé et présent de la théorie anthropologique du didactique. 2007. Disponible en: <http://yves.chevallard.free.fr>. Acceso en: 02 oct. 2014.

CHEVALLARD, Y. Quel programme pour l'avenir de la recherche en TAD. In: CONGRESO INTERNACIONAL SOBRE LA TAD, $3^{\text {th }}, 2010$, Sant Hilari Sacalm. Actas... Barcelona: CRM Documents, 2011, v. 1 p. 23-32. 
CHEVALLARD, Y. Journal du Seminaire TAD/IDD. Théorie Anthropologique du Didactique \& Ingénierie Didactique du Développement.. 2013a. Disponible en :

<ttp://yves.chevallard.free.fr/spip/spip/IMG/pdf/journal-tad-idd-2012-2013-3.pdf>. Acceso en: 02 oct. 2014.

CHEVALLARD, Y. Éléments de didactique du développement durable. Leçon 3. Disponible en: <http://yves.chevallard.free.fr/spip/spip/IMG/pdf/journal-tad-idd-2012-2013-3.pdf〉. Acceso en: 02 oct. 2014.

CORICA, A.; OTERO, M. La formación de profesores de Matemática desde la Teoría Antropológica de lo Didáctico: un estudio de caso. Perspectiva Educacional, v. 53, n. 2, p. 20 - 44, 2014.

CORICA, A.; OTERO, M. The Mathematics Teacher's Profession: the Perspective of Future.

European Journal of Science and Mathematics Education, v. 3, n. 2, p. 145-158. abr. 2015.

DOUADY, R. Dialectique outil-objet et jeux de cadres. Recherches en Didactique des

Mathématiques, Grenoble, v. 7 (2), p. 5-31, 1988.

GASCÓN, J. Incidencia del modelo epistemológico de las matemáticas sobre las prácticas docentes.

RELIME, Ciudad de México, v. 4, n. 2, p. 129-160. jul. 2001.

HERNÁNDEZ, R.; FERNÁNDEZ, C; BAPTISTA, M. Metodología de la investigación. México: Mc Graw Hill, 2010

MINISTERIO DE EDUCACIÓN Y CULTURA. Módulo 4. Estrategias y dispositivos que movilizan. Para potenciar el protagonismo del estudiante. Área de Historia y Geografía. Asunción: MEC, 2011.

PALACIO, L. Reflexiones sobre el texto escolar como dispositivo. Revista Educación y Pedagogía, Medellín, v. 10, n. 21, p. 217-236, may./ago. 1998.

PARRA, V.; OTERO, M. Praxeologías didácticas en la universidad y el fenómeno del encierro: un estudio de caso relativo al límite y continuidad de funciones. In: CONGRESO INTERNACIONAL SOBRE LA TAD, $3^{\text {th }}, 2010$, Sant Hilari Sacalm. Actas... Barcelona: CRM Documents, 2011, v. 1 p. 719-742.

PARRA, V.; OTERO, M.; FANARO, M. Los Recorridos de Estudio e Investigación en la escuela secundaria: resultados de una implementación. Bolema, Rio Claro, v. 27, n. 47, p. 847-874, dic. 2013.

RUIZ, A.; SIERRA, T.; BOSCH, M. Y.; GASCÓN, J. Las Matemáticas para la Enseñanza en una Formación del Profesorado Basada en el Estudio de Cuestiones. Bolema, Rio Claro, v. 28, n. 48, p. 319-340, abr. 2014.

SOUTO, M.; BARBIER, J.; CATTANEO, M.; CORONEL, M.; GAIDULEWICZ, L.; GOGGI, N.; MAZZA, D. Grupos y dispositivos de formación. Buenos Aires: Novedades Educativas, 1999. 